\title{
Gynaecological health in women with delusional disorder: A cross-sectional and one-year follow-up study
}

\author{
Alexandre González-Rodríguez, Oriol Molina-Andreu, Rafael Penadés, María Luisa I maz \\ Gurrutxaga, Rosa Catalán
}

Department of Psychiatry and Psychology. Clinical Institute of Neurosciences. Hospital Clinic, University of Barcelona. Barcelona, Spain.

Correspondence: Alexandre González-Rodríguez. Address: Department of Psychiatry and Psychology. Clinical Institute of Neurosciences. Hospital Clinic, University of Barcelona. Barcelona, Spain. Email: alexgonzalezrguez@gmail.com

Received: December 9, 2013

DOI : $10.5430 /$ jnep.v4n4p81

Accepted: January 1, $2014 \quad$ Online Published: January 20, 2014

URL: http://dx.doi.org/10.5430/jnep.v4n4p81

\section{Abstract}

Introduction: For many decades, clinical and epidemiological associations between gynaecological illness and psychiatric disorders have been reported. Thus, in recent years, special attention has been paid to the gynaecological health of women with chronic psychosis. We aimed to determine the rates of oestrogen and non-oestrogen dependent gynaecological disorders in women diagnosed with delusional disorder (DwDD), and its relationship with clinical outcomes.

Methods: We conducted a longitudinal and observational study in 90 women DwDD who attended our Department of Psychiatry, from 2000 until 2013. Subjects were selected by consecutive sampling and followed-up for one year. The presence or absence of inflammatory and non inflammatory-gynaecological disorders was recorded according to ICD-10 criteria, and attendance rates at appointments (psychiatric and nursing) were collected. For comparisons, $\mathrm{T}$ and Chi-square tests were performed. Multinomial logistic regression was applied to investigate multivariate differences.

Results: Thirty-six (40\%) women DwDD had a history of gynaecological disease (oestrogen dependent disorders: $36.7 \%$ ). Women diagnosed with a gynaecological disease had higher unemployment rates than those without gynaecological conditions $(p=.03)$. Patients without gynaecological disorders attended later our psychiatric service and were more frequently admitted $(p=.048)$. Within the gynaecological group, leiomyoma of uterus $(\mathrm{n}=9 ; 10 \%)$ was the most common condition followed by misscarriage $(n=6 ; 6.7 \%)$. Hysterectomy $(n=7 ; 7.8 \%)$ was the most common surgical treatment. After one year, women diagnosed with gynaecological illness showed a tendency to lower rates of affective comorbidity and less antidepressant prescription. After controlling for age at first appointment and admissions, differences in affective comorbidity remained significant $(p=.020)$; however, differences in antidepressant prescription and employment status were no longer significant.

Conclusions: Women DwDD might be at particular risk of non-inflammatory oestrogen dependent gynaecological disorders. The role of mental health nurses should be addressed to improve attendance rates at gynaecological appointments and prevention programs.

\section{Key words}

Delusional disorder, Paranoia, Gender, Gynaecological health, Gynaecologics 


\section{Introduction}

For many decades, clinical and epidemiological associations between gynaecological illness and psychiatric disorders have been widely reported in the scientific literature.

For instance, in the last century, Bragg ${ }^{[1]}$ analyzed the total admission rates, mental rates for psychoses and readmission rates after hysterectomy or cholecystectomy in Massachusetts. The author found no differences between the observed and expected admission rates for psychoses in the hysterectomy group, and concluded that emotional reactions were more common following hysterectomy when compared to cholecystectomy, although these women did not require admission mental illness.

On the other hand, Barker ${ }^{[2]}$ carried out a case-control study on two clinical groups of women who had undergone a hysterectomy or a cholecystectomy. Within the first two years after hysterectomy, a higher observed incidence of first psychiatric referral was shown when comparing with expected incidence in these patients. Within the patients receiving cholecystectomy, no statistically significant differences were found between expected and observed incidences of psychiatric referrals.

Overall, the role of consulting psychiatrists in a tertiary general hospital has been emphasized. In a retrospective study on clinical records ${ }^{[3]}$, nearly $30 \%$ of the evaluated gynaecologic outpatients had a diagnosis of a psychiatric disorder, the most common being depressive disorders, followed by schizophrenia and other psychoses.

Several psychiatrists and gynaecologists have also confirmed the clinical impression that gynaecological symptoms are significantly associated with diagnosis of psychiatric disorders. In a community survey in Oxford ${ }^{[4]}$, although no associations between psychiatric disorders and gynaecological health were found, mood disturbances were associated with oestrogen-deficit conditions.

According to these findings, some authors ${ }^{[5-8]}$ have suggested that gonadal dysfunction and oestrogen deficits would be implicated in the etiology of psychotic disorders. Two hypotheses have been proposed: "the oestrogen protection hypothesis" and "the hypothesis of hypoestrogenism". In the last decades, many epidemiological and life-cycle studies have supported the "oestrogen protection hypothesis". Within first-episode of psychosis patients, men had an earlier age at onset of the illness, while women had a second spike of incidence between the fourth and fifth decades ${ }^{[6]}$. Furthermore, Bergemann and coworkers ${ }^{[9]}$ evaluated the effect of menstrual cycle or oestrogen levels on psychotic symptoms in a sample of women diagnosed with schizophrenia, and found a significant improvement in symptoms during the luteal phase when compared to low-oestrogen follicular phases.

On the other hand, several reviews have strongly supported the "hypothesis of the hypoestrogenism" ${ }^{[7,10]}$. Women diagnosed with schizophrenia had menstrual irregularities, fewer pregnancies and physical signs of hyperandrogenism, and lower levels of oestrogens than women without a diagnosis of schizophrenia.

As suggested by previous reports ${ }^{[1]}$, many disturbances of genital function such as dysmenorrhoea and metrorrhagia can be found in women with low social support and comorbid psychiatric symptoms.

When focusing on the gynaecological health of psychotic women at the beginning of the XXth century, gynaecologists reported that about $50 \%$ of female patients diagnosed with schizophrenia, 1/3 of women diagnosed delusional disorder and dementia had a gynaecological disease, which was more common than in healthy women ${ }^{[12]}$.

A recent systematic review ${ }^{[13]}$ emphasized that prevalence rates of breast and cervical cancer would be a useful indicator to better understand the impact of severe mental illness on these gynaecological disorders, and to develop effective interventions to prevent them. In this line, Barak and coworkers ${ }^{[14,15]}$ found a significant reduced risk of breast cancer in a tertiary care group of female patients diagnosed with schizophrenia. However, the authors concluded that breast and ovarian cancer screening should be taken into account, specifically with weight gain or hyperprolactinemia in women 
diagnosed with schizophrenia. By contrast, a meta-analysis of observational clinical studies on female patients diagnosed with schizophrenia concluded that breast cancer may be increased in these patients, but several methodological problems were found in the reported studies ${ }^{[16]}$. Due to these controversial findings, the authors also considered that breast cancer screening should be emphasized in female patients diagnosed with schizophrenia.

Regarding the screening of gynaecological cancers, several studies have reported that women diagnosed with schizophrenia received mammograms less frequently and attended fewer to gynaecological services than other women ${ }^{[17,18]}$. Lindamer and co-workers ${ }^{[19]}$ confirmed that low adherence to mammography screening are indeed frequent among these women and recommended additional measures to increase breast cancer screening rates. Moreover, a recent study found that women diagnosed with psychosis were less likely to receive Pap screening for cervical cancer in comparison with women from the general population ${ }^{[20]}$.

It has been well established that some common gynaecological disorders have an oestrogen-dependent pattern. For instance, adenomyosis and endometriosis have been reported to be more prevalent in women at a reproductive age and less frequent in the menopause years ${ }^{[21]}$. These oestrogen-dependent conditions have been associated with infertility at lifetime; however, few studies have investigated the relationship between oestrogen-dependent gynaecological illness and psychiatric disorders.

Our goal was to determine the rates of inflammatory and non-inflammatory gynaecological disorders (oestrogen and non-oestrogen dependent disease) in female patients DwDD, and to relate those rates with demographic and clinical data.

Gender differences in attendance rates for appointments in DD have been widely studied; however, samples of women DwDD have not been divided by gynaecological illness ${ }^{[22]}$. Thus, we also aimed to investigate whether women DwDD and gynaecological illness and women without such conditions differ in attendance rates at medical and nursing psychiatric appointments at a one year follow-up. Our point when addressing attendance was to measure, albeit in an indirect fashion, if poorer self-care protrayed some role in the manifestation of these medical conditions in our patients.

To our knowledge, no studies have explored the clinical correlations of gynaecological disorders and related conditions, and psychiatric symptoms in women DwDD.

\section{Methods}

\subsection{Participants and study design}

We conducted a longitudinal and naturalistic study in female patients DwDD, who were attended at our Department of Psychiatry and Psychology, from January 2000 until January 2013. The subjects were selected by consecutive sampling and followed-up for one year. Our work is a representative study of all first attended patients with delusional disorder from a well-defined catchment area with a population of about 250.000 inhabitants in Barcelona. This seems to be representative, as our Schizophrenia and related disorders Program is of reference in Barcelona.

All subjects fulfilled the following eligibility criteria: (1) aged over 18 years and (2) met DSM-IV-TR criteria for DD. Patients previously diagnosed as having schizophrenia, organic psychosis or mental retardation were excluded.

The present study is a part of an ongoing study on schizophrenia and related disorders, which was approved by the Ethics Committee of our hospital (Ref.20073699), and written informed consent was obtained from each patient.

\subsection{Measures}

Sociodemographic and clinical variables at the time of the study inclusion were obtained, including age at onset of DD, age at first psychiatric appointment, marital status, educational level, employment status, number of children and 
cohabiters, reasons leading to consultation, DD type, presence/absence of non-prominent hallucinations, depressive co-morbidity, and suicidal ideation and suicidal behaviour at lifetime.

At baseline, social functioning was also assessed by using the Global Assessment of Functioning Scale (GAF), which is a numeric scale (0 through 100) used by mental health clinicians and physicians to rate subjectively the social, occupational, and psychological functioning of adults. The scale is presented and described in the DSM-IV-TR on page $34^{[23]}$.

Operationalized definitions were established to assess compliance at medical and nursing psychiatric appointments during the follow-up period. Patients were classified as follows: patients who discontinued appointments before 6 months, between 6 and 12 months, and after 12 months from the first psychiatric appointment. Furthermore, patients were grouped into three groups attending to the compliance rates at appointments: those who kept $<25 \%$ of the scheduled appointments, between $25 \%$ and $75 \%$, and those who attended $>75 \%$ of appointments during their period of contact.

The presence or absense of gynaecological diseases in these patients were obtained by questioning patients and reviewing medical records, and were coded according to ICD-10 criteria. Gynaecological illness was classified as follows: (1) non-inflammatory oestrogen dependent diseases, (2) non-inflammatory non-oestrogen dependent and (3) inflammatory diseases. Also, we recorded the following gynaecologic surgical treatments: uterine polypectomy, uterine myomectomy, uterine curettage, hysterectomy, unilateral oophorectomy, bilateral oophorectomy, salpingectomy and breast mastectomy.

Admission rates at a psychiatric inpatient clinic, antidepressant prescription rates, attendance rates at medical and nursing psychiatric appointments, and the frequency of appointments attended were defined as outcome variables. Other clinical variables were considered secondary outcomes.

Demographic and clinical data, as well as gynaecological variables were obtained from all participants of the study. In a second step, the total sample was divided into two groups according to the presence of gynaecological disorders: (1) women DwDD with gynaecological disorders and (2) women DwDD without these conditions.

\subsection{Statistical analysis}

The SPSS version 19.0 software (SPSS Inc., Chicago, Illinois, USA) was used to perform the statistical analyses. Univariate comparisons in socio-demographic and clinical characteristics between female patients DwDD with and without diagnoses of gynaecological diseases were tested with $t$-tests, Mann-Whitney $U$ tests, $\chi^{2}$ and Fisher's exact tests. Multinomial logistic regression was applied to investigate multivariate differences. Affective comorbidity, antidepressant prescription, employment status and attendance rates at appointments served as the dependent variables, age at first psychiatric appointment and number of admissions were included into the model as confounding factors, and clinical group (gynaecological disease group and non-gynaecological disease) appeared in the model as a fixed factor.

\section{Results}

\subsection{Sample characteristics}

From 2000 until 2013, 117 women DwDD were attended at our Department of Psychiatry. Ninety patients accepted to participate in the study and underwent a systematic demographic and clinical questionnaire, including questions about their gynaecological health.

Thirty-six (40\%) out of the ninety women DwDD included in the study, had a history of gynaecological disease diagnosis, the most common were non-inflammatory oestrogen dependent disorders (36.7\%). 
Table 1. Sociodemographic and clinical characteristics of women DwDD by clinical groups

\begin{tabular}{|c|c|c|c|c|}
\hline & $\begin{array}{l}\text { Total } \\
(\mathrm{N}=90)\end{array}$ & $\begin{array}{l}\text { Gynaecological disease } \\
(n=36)\end{array}$ & $\begin{array}{l}\text { Non-gynaecological } \\
\text { disease }(n=54)\end{array}$ & Statistic \\
\hline \multicolumn{5}{|l|}{ Sociodemographic and clinical measures } \\
\hline \multicolumn{5}{|l|}{ Marital status $[\mathrm{n}(\%)]$} \\
\hline Single & $35(38.9)$ & $13(36.11)$ & $22(40.74)$ & \multirow{4}{*}{$\begin{array}{l}\chi^{2}=0.404, \mathrm{df}=3, \\
p=.939\end{array}$} \\
\hline Married/living together & $23(25.6)$ & $10(27.78)$ & $13(24.07)$ & \\
\hline Separated/divorced & $26(28.9)$ & $11(30.56)$ & $15(27.78)$ & \\
\hline Widowed & $6(6.7)$ & $2(5.56)$ & $4(7.41)$ & \\
\hline \multicolumn{5}{|l|}{ Educational level, years [n (\%)] } \\
\hline$<8$ & $18(20)$ & $4(11.11)$ & $14(25.93)$ & \multirow{4}{*}{$\begin{array}{l}\chi^{2}=5.921, \mathrm{df}=3, \\
p=.116\end{array}$} \\
\hline $8-9$ & $33(36.7)$ & $12(33.33)$ & $21(38.89)$ & \\
\hline $10-11$ & $20(22.2)$ & $12(33.33)$ & $8(14.81)$ & \\
\hline 12 or $>$ & $19(21.1)$ & $8(22.22)$ & $11(20.37)$ & \\
\hline \multicolumn{5}{|l|}{ Employment status [n (\%)] } \\
\hline Unemployed & $20(22.2)$ & $13(36.11)$ & $7(12.96)$ & \multirow{3}{*}{$\begin{array}{l}\chi^{2}=7.016, \mathrm{df}=2, \\
p=.030 *\end{array}$} \\
\hline Employed & $33(36.7)$ & $12(33.33)$ & $21(38.89)$ & \\
\hline Pensioner & $37(41.1)$ & $11(30.56)$ & $26(48.14)$ & \\
\hline Number of children, mean (SD) & $0.90(1.09)$ & $1.03(1.28)$ & $0.81(0.95)$ & $\begin{array}{l}\mathrm{T}=-0.906, \mathrm{df}=88, \\
p=.368\end{array}$ \\
\hline Number of cohabiters, mean (SD) & $0.69(0.96)$ & $0.56(0.69)$ & $0.78(1.09)$ & $\begin{array}{l}\mathrm{T}=1.179, \mathrm{df}=87.85, \\
p=.242\end{array}$ \\
\hline Age at onset of DD, mean (SD) & $50.96(12.22)$ & $49.49(12.62)$ & $51.91(11.97)$ & $\begin{array}{l}\mathrm{T}=0.913, \mathrm{df}=87 \\
p=.364\end{array}$ \\
\hline $\begin{array}{l}\text { Age at first psychiatric appointment, mean } \\
\text { (SD) }\end{array}$ & $56.17(13.49)$ & $53.39(12.9)$ & $58.02(13.68)$ & $\begin{array}{l}\mathrm{T}=1.609, \mathrm{df}=88, \\
p=.111\end{array}$ \\
\hline GAF scores, mean (SD) & $40.97(10.42)$ & $42.06(12.18)$ & $40.24(9.11)$ & $\begin{array}{l}\mathrm{T}=-0.763, \mathrm{df}=88, \\
p=0.448\end{array}$ \\
\hline \multicolumn{5}{|l|}{ Reasons leading to consultation [n (\%)] } \\
\hline Delusional thought & $21(23.3)$ & $7(19.44)$ & $14(25.93)$ & \multirow{5}{*}{$\begin{array}{l}\chi^{2}=6.318, \mathrm{df}=4, \\
p=.177\end{array}$} \\
\hline Behavioural disorders & $51(56.7)$ & $18(50)$ & $33(61.11)$ & \\
\hline Anxiety & $9(10)$ & $7(19.44)$ & $2(3.70)$ & \\
\hline Depressive symptoms & $2(2.2)$ & $1(2.78)$ & $1(1.85)$ & \\
\hline Suicidality & $7(7.8)$ & $3(8.33)$ & $4(7.41)$ & \\
\hline \multicolumn{5}{|l|}{ DD type $[\mathrm{n}(\%)]$} \\
\hline Persecutory & $66(73.3)$ & $27(75)$ & $39(50)$ & \multirow{6}{*}{$\begin{array}{l}\chi^{2}=5.484, \mathrm{df}=5, \\
p=.360\end{array}$} \\
\hline Erotomanic & $9(10)$ & $4(11.11)$ & $5(9.26)$ & \\
\hline Grandiose & $2(2.2)$ & $0(0)$ & $2(3.70)$ & \\
\hline Somatic & $7(7.8)$ & $1(2.78)$ & $6(11.11)$ & \\
\hline Jealous & $4(4.4)$ & $3(8.33)$ & $1(1.85)$ & \\
\hline Mixed & $2(2.2)$ & $1(2.78)$ & $1(1.85)$ & \\
\hline \multicolumn{5}{|l|}{ Presence of hallucinations [n (\%)] } \\
\hline Olfactory & $7(7.8)$ & $2(5.56)$ & $5(9.26)$ & \multirow{4}{*}{$\begin{array}{l}\chi^{2}=7.080, \mathrm{df}=4, \\
p=.132\end{array}$} \\
\hline Tactil & $7(7.8)$ & $0(0)$ & $7(12.96)$ & \\
\hline Non-prominent auditive & $12(13.3)$ & $5(13.89)$ & $7(12.96)$ & \\
\hline Non-prominent visual & $1(1.1)$ & $1(2.78)$ & $0(0)$ & \\
\hline $\begin{array}{l}\text { Depressive comorbidity [n (\%)] } \\
\text { Presence }\end{array}$ & $31(34.4)$ & $10(27.78)$ & $21(38.89)$ & $p=.051 ;$ FET \\
\hline Suicidal ideation at lifetime [n (\%)] & $19(21.1)$ & $7(19.44)$ & $12(22.22)$ & $p=.798 ; \mathrm{FET}$ \\
\hline Suicidal behaviour at lifetime [n (\%)] & $11(12.2)$ & $3(8.33)$ & $8(14.81)$ & $p=.515 ;$ FET \\
\hline \multicolumn{5}{|l|}{ Outcome variables } \\
\hline Antidepressants at follow-up [n (\%)] & $58(64.44)$ & $26(72.22)$ & $32(59.26)$ & $\begin{array}{l}\chi^{2}=10.785, \mathrm{df}=5, \\
p=.056\end{array}$ \\
\hline Number of admissions [n (\%)] & $0.41(0.685)$ & $0.25(0.50)$ & $0.52(0.771)$ & $\begin{array}{l}\mathrm{T}=2.005, \mathrm{df}=88, \\
p=.048^{*}\end{array}$ \\
\hline \multicolumn{5}{|l|}{ Maintenance rates at follow-up, [n (\%)] } \\
\hline$<6$ months & $23(25.56)$ & $8(25)$ & $15(30)$ & \multirow{3}{*}{$\begin{array}{l}\chi^{2}=0.482, \mathrm{df}=2, \\
p=.786\end{array}$} \\
\hline 6-12 months & $9(10)$ & $3(9.4)$ & $6(12)$ & \\
\hline$>12$ months & $50(55.56)$ & $21(65.6)$ & $29(58)$ & \\
\hline
\end{tabular}


Sociodemographic and clinical characteristics of women DwDD by clinical groups are shown in Table 1.

No statistically significant differences were found between women DwDD with gynaecological diseases and those without them regarding years of education, marital status, and number of children and cohabiters. However, women without a history of gynaecological illness were more likely to be employed than female DwDD and comorbid gynecological conditions $(p=.03)$.

There were no statistically significant differences in age at onset of DD, reasons leading to consultation, DD type, presence of hallucinations, and presence of suicidal ideation and lifetime suicidal behaviour.

Although no statistical significance was reached, when compared to women DwDD without gynaecological illnesses, female patients DwDD and gynaecological diseases attended earlier our outpatient clinic [53.39 (12.9) vs. 58.02 (13.68)] and showed a tendency to have lower rates of affective comorbidity.

\subsection{Rates of gynaecological diseases and gynaecologic surgical treatments}

Classifications of gynaecological diseases and gynaecologic surgical treatments in female DwDD patients of our sample are presented in Table 2.

Table 2. Classification of gynaecological diseases and gynaecologic surgical treatments in women with delusional disorder $(\mathrm{N}=90)$

\begin{tabular}{|c|c|}
\hline ICD-10 Code- Gynaecological disease & $\mathbf{N}(\%)$ \\
\hline Non-inflammatory gynaecological disease & $33(36.67)$ \\
\hline C50- Breast cancer & $5(5.56)$ \\
\hline C55- Endometrial Cancer & $1(1.11)$ \\
\hline C56- Ovarian Cancer & $1(1.11)$ \\
\hline D25- Leiomyoma of uterus & $9(10)$ \\
\hline E28.2- Polycystic ovarian syndrome & $2(2.22)$ \\
\hline E28.3- Primary ovarian failure (Premature menopause) & $1(1.11)$ \\
\hline N60.2- Breast adenoma & $1(1.11)$ \\
\hline N83.20- Ovarian cyst & $2(2.22)$ \\
\hline N85.0- Endometrial polyp & $4(4.44)$ \\
\hline N85.01- Bening endometrial hyperplasia & $1(1.11)$ \\
\hline N92- Excessive frequent and irregular menstruation (at lifetime) & $5(5.56)$ \\
\hline N96.0- Recurrent miscarriage & $1(1.11)$ \\
\hline Q00- Ectopic pregnancy & $2(2.22)$ \\
\hline Q03-Miscarriage & $6(6.67)$ \\
\hline Inflammatory gynaecological disease & $5(5.56)$ \\
\hline A63- Condylomaacuminatum & $1(1.11)$ \\
\hline N60.1- Diffuse cystic mastopathy & $1(1.11)$ \\
\hline N76.3- Subacute and chronic vulvitis & $1(1.11)$ \\
\hline N77.1- Candidiasis of vagina & $1(1.11)$ \\
\hline Q91.12- Breast abscess & $1(1.11)$ \\
\hline Gynaecologic surgical treatments & $23(25.56)$ \\
\hline Uterine polypectomy & $4(4.44)$ \\
\hline Uterine myomectomy & $1(1.11)$ \\
\hline Uterine curettage & $2(2.22)$ \\
\hline Hysterectomy & $7(7.78)$ \\
\hline Unilateral oophorectomy & $1(1.11)$ \\
\hline Bilateral oophorectomy & $2(2.22)$ \\
\hline Unilateral salpingectomy & $1(1.11)$ \\
\hline Breast mastectomy & $5(5.56)$ \\
\hline
\end{tabular}

Note. Abbreviations: ICD-10, International Statistical Classification of Diseases 10th revision. 
Within the gynaecological disease group, the most common condition was leiomyoma of uterus ( $\mathrm{n}=9 ; 10 \%)$, followed by misscarriage $(n=6 ; 6.7 \%)$, excessive frequent and irregular menstruations at lifetime $(n=5 ; 5.56 \%)$ and breast cancer $(n$ $=5 ; 5.56 \%)$.

Twenty-six per cent of the sample had a history of gynaecologic surgical treatment. Hysterectomy $(\mathrm{n}=7 ; 7.78 \%)$ was the most frequently encountered, followed by breast mastectomy $(n=5 ; 5.56 \%)$ and uterine polypectomy $(n=4 ; 4.4 \%)$. Other surgical conditions were less frequent.

\subsection{Comparisons in outcome variables by clinical groups}

Descriptives and comparisons in outcome variables are also presented in Table 2 and Figure 1.

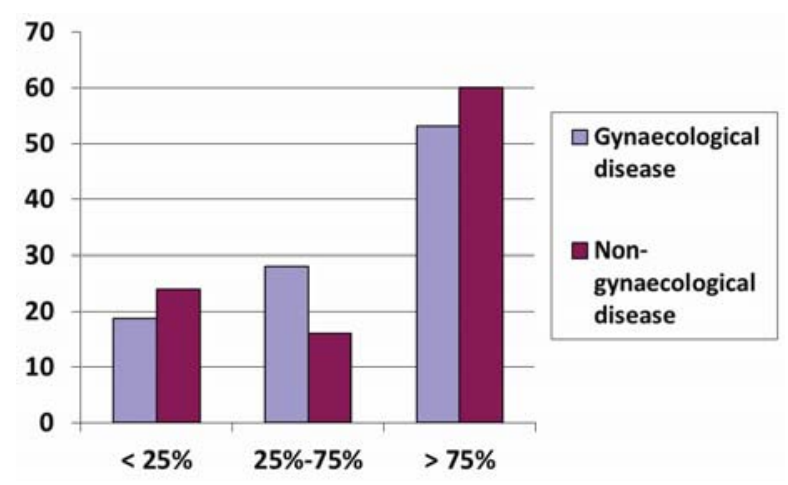

Figure 1. Percentages of attendance at psychiatric and nursing appointments at follow-up by clinical group

When uncorrected for potential confounders, patients without a history of gynaecological disease were more frequently admitted at our psychiatric inpatient unit during the follow-up period $(p=.048)$ in comparison DwDD women without gynaecological conditions. However, no statistically significant differences were found between both groups in terms of maintenance rates at follow-up $(p=.786)$ and percentages of attendance at psychiatric and nursing appointments $(p=$ $.409)$.

On the other hand, although no statistical significance was reached, women diagnosed and with gynaecological disorders showed a tendency to more frequently receive antidepressants than those without such conditions (72.22\% vs 59.26).

In a second step, we performed Multinomial Logistic Regression analyses in which affective comorbidity, antidepressant prescription, employment status, attendance rates at appointments and maintenance at follow-up were included as dependent variables.

After adjustment for potential confounders, differences in affective comorbidity remained significant $\left(\chi^{2}=4.647 ; p=\right.$ $.031)$; however, differences in antidepressant prescription $\left(\chi^{2}=1.528 ; p=.216\right)$ and employment status $\left(\chi^{2}=5.514 ; p=\right.$ $.063)$ were no longer significant. Furthermore, we found that the presence or absence of gynaecological disorders did not predict statistically significant differences in terms of percentages of maintenance rates $\left(\chi^{2}=0.615 ; p=.735\right)$ and attendance rates for appointments $\left(\chi^{2}=1.901 ; p=.387\right)$.

\section{Discussion}

We studied 90 DD women who attended our Department of Psychiatry and Psychology over a period of ten years. We carried out cross-sectional comparisons at baseline and followed-up them for one year. 
Thirty-six (40\%) of these female patients DwDD showed a history of gynaecological diseases. To date, this is the first study to examine the gynaecological health of women DwDD, and to relate it to clinical outcomes.

These findings are in agreement with classical authors ${ }^{[12]}$ who reported that nearly $47 \%$ of women diagnosed with psychiatric disorders also have some diagnosed gynaecological disorders in pelvic organs. The authors, in a second step, analyzed the distribution of these lesions among the different forms of psychiatric disorders, and found that $50 \%$ of psychotic women had a gynaecological illness. Furthermore, our findings are supported by recent findings by Lindamer and co-workers ${ }^{[17,19]}$ who found that women diagnosed with schizophrenia or chronic psychoses received fewer gynaecological services, and gynaecological disease was the most commonly diagnosed medical problem.

However, no recent studies are available regarding the prevalence diagnoses of gynaecological disorders in women DwDD.

In a first sample description, we classified gynaecological disorders in three groups according to the most important groups described in ICD-10: non-inflammatory oestrogen dependent diseases, non-inflammatory non-oestrogen dependent diseases and inflammatory illnesses. We found that oestrogen dependent disorders were the most common gynaecological conditions encountered (36.7\%), whereas a minority of patients showed inflammatory disorders. These results are also in line with the study mentioned above that reported low rates of inflammatory troubles in the entire sample ${ }^{[12]}$.

In our sample, the most common diagnosed gynaecological condition was leiomyoma of uterus, followed by miscarriage and excessive frequent and irregular menstruations at lifetime. These findings are consistent with previous reviews ${ }^{[6,10]}$, which reported that psychotic women might have lower pregnancies and higher infertility rates than healthy women, probably as a result of gonadal dysfunction and oestrogen deficits. Our study supports the hypothesis of hypoestrogenism in women diagnosed with chronic psychotic disorders ${ }^{[6]}$.

Regarding gynaecologic surgical treatment in our sample, hysterectomy was the most frequently encountered (7.78\%), followed by breast mastectomy (5.56\%) and uterine polypectomy (4.4\%). No studies have examined the rates of surgical treatments and their gynaecological comorbidity in women DwDD. Within patients diagnosed with chronic schizophrenia, Lindamer and colleagues ${ }^{[17]}$ found that hysterectomy was the most frequent surgical treatment in these women $(29 \%)$. However, our sample was not restricted in terms of age, and premenopausal women were also included into the analysis.

In a second step, we divided the sample into two groups according to the presence or absence of gynaecological disorders. We found that women DwDD with a history of gynaecological illness were more likely to be unemployed than those without such conditions, which suggests that medical conditions should be integrated in a multifactorial model to better explain social and personal functioning in women diagnosed with psychosis. The possibility of comparing these results with previous studies does not exist due to the lack of clinical studies on these populations.

When uncorrected for influencing factors, several clinical variables such as age at onset of DD, reasons leading to consultation, DD type, presence of hallucinations, and presence of suicidal ideation or behavior did not differ between DD women with gynaecological illness and those without them. However, female patients DwDD and gynaecological diseases showed a tendency to have lower rates of affective comorbidity, an earlier age at the first psychiatric appointment, and showed lower rates of psychiatric admissions in comparison DwDD women without a history of gynaecological illness.

A recent study ${ }^{[22]}$ has found that women DwDD attended first time psychiatric appointments later than men. However, previous studies have not taken into account the influence of other medical problems, such as gynaecological diseases, on the attendance rates at psychiatric clinics. For this reason, we aimed to investigate whether women DwDD with or without gynaecological problems differ in attendance rates for medical or nursing psychiatric appointments. Prior to the correction 
for confounding factors, we found no statistically significant differences in maintenance rates at the follow-up period nor in percentages of attendance at the psychiatric appointments.

In a second step, we included age at first psychiatric appointment and number of admissions into the model as confounding factors, affective comorbidity, antidepressant prescription, employment status and attendance rates at appointments as dependent variables, and clinical group (gynaecological disease group and non-gynaecological disease) as a fixed factor.

After adjustment, differences in affective comorbidity remained significant; but differences in antidepressant prescription and employment status were no longer significant. No statistically significant differences were found in attendance rates for appointments and maintenance rates at follow-up between both groups.

In our study, several limitations should be taken into account: (1) the small sample size that could have limited the statistical power, (2) lack of data on the prevalence of gynaecological illness in women DwDD who did not attend our psychiatric clinic, and (3) the study design that have limited the possibility to study the role of the loss of ovarian function in the psychopathology of women DwDD. However, some strength should be noted. To the best of our knowledge, this is the first study to specifically describe sociodemographic and clinical factors that may be implicated in the clinical course of women DwDD with or without gynaecological disorders. Furthermore, to date, no studies have investigated the rates of non-inflammatory and inflammatory gynaecological disorders in a clinical group of women DwDD.

\section{Conclusions}

Women DwDD might be at particular risk of non-inflammatory oestrogen dependent gynaecological disorders and may require earlier attendance at our outpatient clinic. We emphasize the role of mental health nurses in the follow-up of these patients, and we propose the integration of nurses in specific prevention programs in women diagnosed with psychiatric disorders.

\section{References}

[1] Bragg RL. Risk of Admission to Mental Hospital Following Hysterectomy or Cholecystectomy. Am J Public Health Nations Health. 1965; 55: 1403-1410. http://dx.doi.org/10.2105/AJPH.55.9.1403

[2] Barker MG. Psychiatric illness after hysterectomy. Br Med J. 1968; 2: 91-95. http://dx.doi.org/10.1136/bmj.2.5597.91

[3] Lin HL, Chou HH, Liu CY, Hsu SC, Hsiao MC, Juang YY. The role of consulting psychiatrists for obstetric and gynecologic inpatients. Chang Gung Med J. 2011; 34: 57-64.

[4] Gath D, Osborn M, Bungay G, Iles S, Day A, Bond A, Passingham C. Psychiatric disorder and gynaecological symptoms in middle aged women: a community survey. Br Med J (Clin Res Ed). 1987; 294: 213-218. http://dx.doi.org/10.1136/bmj.294.6566.213

[5] Mahé V, Dumaine A. Oestrogenwithdrawal associated psychoses. ActaPsychiatr Scand. 2001; 104: 323-331. http://dx.doi.org/10.1034/j.1600-0447.2001.00288.x

[6] Riecher-Rössler A, Seeman MV. Oestrogens and schizophrenia--introduction. Arch WomensMent Health. 2002 ; 5: $91-92$. http://dx.doi.org/10.1007/s007370200026

[7] Riecher-Rössler A. Oestrogen effects in schizophrenia and their potential therapeutic implications--review. Arch WomensMent Health. 2002; 5: 111-118. http://dx.doi.org/10.1007/s00737-002-0003-3

[8] Hayes E, Gavrilidis E, Kulkarni J. The Role of Oestrogen and Other Hormones in the Pathophysiology and Treatment of Schizophrenia. Schizophr Res Treatment. 2012; 2012: 540273. http://dx.doi.org/10.1155/2012/540273

[9] Bergemann N, Parzer P, Runnebaum B, Resch F, Mundt C. Estrogen, menstrual cycle phases, and psychopathology in women suffering from schizophrenia. Psychol Med. 2007; 37: 1427-1436.

[10] Riecher-Rössler A, Kulkarni J. Estrogens and gonadal function in schizophrenia and related psychoses. Curr Top BehavNeurosci. 2011; 8: 155-171.

[11] Snaith L, Ridley B. Gynaecological psychiatry; a preliminary report on an experimental clinic. Br Med J. 1948; 2: $418-421$. http://dx.doi.org/10.1136/bmj.2.4573.418 
[12] Taussig FJ. Gynecologic disease in the insane and its relationship to the various forms of psychoses. JAMA. 1912; LIX: 713-716. http://dx.doi.org/10.1001/jama.1912.04270080395007

[13] Aggarwal A, Pandurangi A, Smith W. Disparities in breast and cervical cancer screening in women with mental illness: a systematic literature review. Am J Prev Med. 2013; 44: 392-398. http://dx.doi.org/10.1016/j.amepre.2012.12.006

[14] Barak Y, Achiron A, Mandel M, Mirecki I, Aizenberg D. Reduced cancer incidence among patients with schizophrenia. Cancer. 2005; 104: 2817-2821. http://dx.doi.org/10.1002/cncr.21574

[15] Barak Y, Levy T, Achiron A, Aizenberg D. Breast cancer in women suffering from serious mental illness. Schizophr Res. 2008; 102: 249-253. http://dx.doi.org/10.1016/j.schres.2008.03.017

[16] Bushe CJ, Bradley AJ, Wildgust HJ, Hodgson RE. Schizophrenia and breast cancer incidence: a systematic review of clinical studies. Schizophr Res. 2009; 114: 6-16. http://dx.doi.org/10.1016/j.schres.2009.07.012

[17] Lindamer LA, Buse DC, Auslander L, Unützer J, Bartels SJ, Jeste DV. A comparison of gynecological variables and service use among older women with and without schizophrenia. Psychiatr Serv. 2003; 54: 902-904.

[18] Martens PJ, Chochinov HM, Prior HJ, Fransoo R, Burland E; Need To Know Team. Are cervical cancer screening rates different for women with schizophrenia? A Manitoba population-based study. Schizophr Res. 2009; 113: 101-106. http://dx.doi.org/10.1016/j.schres.2009.04.015

[19] Lindamer LA, Wear E, Sadler GR. Mammography stages of change in middle-aged women with schizophrenia: an exploratory analysis. BMC Psychiatry. 2006; 6: 49. http://dx.doi.org/10.1186/1471-244X-6-49

[20] Tilbrook D, Polsky J, Lofters A. Are women with psychosis receiving adequate cervical cancer screening? Can Fam Physician. 2010; 56: 358-363.

[21] Kitawaki J. Adenomyosis: the pathophysiology of an oestrogen-dependent disease. Best Pract Res ClinObstetGynaecol. 2006; 20: 493-502. http://dx.doi.org/10.1016/j.bpobgyn.2006.01.010

[22] González-Rodríguez A, Molina-Andreu O, ImazGurrutxaga ML, Catalán Campos R, Bernardo Arroyo M. A descriptive retrospective study of the treatment and outpatient service use in a clinical group of delusional disorder patients. Rev Psiquiatr Salud Ment, in press.

[23] American Psychiatric Association. Diagnostic and statistical manual of mental disorders (4th ed., text rev.); 2000. Washington, DC: Author. 